\title{
SUSY overview
}

\section{Werner Porod*}

Universität Würzburg, Germany

E-mail: Werner.Porodeuni-wuerzburg.de

We summarize the status of various supersymmetric models in view of the existing LHC data. A particular focus is on the implications of the measured Higgs mass on these models which gives important constraints. We consider here minimal and non-mininal supersymmetric extension of the Standard Model

An Alpine LHC Physics Summit (ALPS2018)

15-20 April, 2018

Obergurgl, Austria

${ }^{*}$ Speaker. 


\section{Introduction}

The discovery of the Higgs boson at the LHC [1,2] marks one of the most important milestones in particle physics. Its mass is known rather precisely: ATLAS reports $m_{h}=124.97 \pm 0.24 \mathrm{GeV}$ [3] and CMS $m_{h}=125.26 \pm 0.21 \mathrm{GeV}$ [4]. Moreover, the signal strength of LHC searches in various channels for both bosonic and fermionic final states has been found consistent with predictions of the Standard Model (SM), see e.g. [5, 6, 7, 8]. Even though this completes the SM particle-wise, several important questions still remain open, e.g. (i) Is it possible to include the SM in a grand unified theory where all gauge forces unify? (ii) What stabilizes the Higgs mass at the electroweak scale? (iii) Is there a particle physics explanation of the observed dark matter relic density?

Supersymmetry (SUSY) is still one of the best motivated extensions of SM addressing several of these questions. Consequently, the search for SUSY is among the main priorities of the LHC collaborations. Up to now no sign for supersymmetry or any significant deviation from the Standard Model (SM) prediction has been found has been found at the $\mathrm{LHC}^{1}$, e.g. in simplified models bounds on the gluino mass of up to about $2 \mathrm{TeV}$ have been set in various channels $[9,10,11,12]$ exploiting about $36 \mathrm{fb}^{-1}$ of data in each experiment. However, note that these bounds depend on the spectrum and get reduced significantly if the spectrum is rather compressed [13] as has been noted early on [14].

In the minimal supersymmetric standard model (MSSM) the mass of the lighter Higgs boson is bounded to be below the mass of the Z-boson at tree level implying the need of very large radiative corrections of at least $90 \%$ with respect to the tree-level as $m_{h}^{2} \simeq m_{Z}^{2}+86^{2} \mathrm{GeV}$. It has been known for a long time that such large radiative corrections due to the top-quark and stops, the scalar partners of the top-quark, indeed exist as the top-Yukawa coupling is order 1 . This requires that either the geometric average of the stop masses $M_{S}^{2}=m_{\tilde{t}_{1}} m_{\tilde{t}_{2}}$ is large and/or the existence of large trilinear coupling $A_{t}$ [22] as can be seen by inspecting the most dominant contributions which are given by

$$
\Delta m_{h}^{2}=+\frac{3 m_{t}^{4}}{4 \pi^{2} v^{2}}\left[\ln \left(\frac{M_{S}^{2}}{m_{t}^{2}}\right)+\frac{X_{t}^{2}}{M_{S}^{2}}\left(1-\frac{X_{t}^{2}}{12 M_{S}^{2}}\right)\right] .
$$

$X_{t}=A_{t}-\mu \cot \beta$ is a measure of the left-right mixing with $\mu$ being the higgsino mass parameter, $\tan \beta=v_{u} / v_{d}$ the ratio of the two vacuum expectation values and $v^{2}=v_{u}^{2}+v_{d}^{2}=4 m_{W}^{2} / g^{2}$.

\section{Implication for models with MSSM particle content}

The question, to which extent the observed Higgs mass can be explained within a given supersymmetric high-scale model and what are the resulting implications on the spectrum has been investigated by several authors. The main results can be summarized as follows: in minimal gauge mediated SUSY breaking (GMSB) models one finds $m_{\tilde{t}_{1}} \gtrsim 6 \mathrm{TeV}$ with $\tilde{t}_{1}$ being the lightest of the coloured SUSY particles als the lightest neutralino is in the TeV range [23]. The main reason is

\footnotetext{
${ }^{1}$ However, there are intriguing hints in at nearly 4 sigma that lepton universality could be violated in $B$-meson decays which would be a clear signal for new physics. This could partially be explained in the context of supersymmetry [15] but might require different extensions, e.g. leptoquarks, see for example [16, 17, 18] or new vector bosons, see for example $[19,20,21]$.
} 
that at the so-called messenger scale one finds $A_{t}=0$ requiring the stops to be very heavy. If this were realized in nature, the LHC at $14 \mathrm{TeV}$ would not be able to discover SUSY but would require a higher center of mass energy, e.g. a $100 \mathrm{TeV}$ collider [24]. However, in extended GMSB models non-zero trilinear couplings are possible at the messenger scale and consequently there are corners in parameter space [25] with lighter squarks, e.g. $m_{\tilde{t}_{1}} \simeq m_{\tilde{b}_{1}} \gtrsim 1 \mathrm{TeV}$, which is the mass range explored by the current LHC run [10,26].

In the constrained MSSM (CMSSM) or slightly extended versions with non-universal Higgs mass parameters (NUHM-models) the explanation of the observed Higgs mass requires at $M_{G U T}$, the scale of grand unification (GUT), the condition $\left|A_{0}\right| \simeq 2 m_{0}[27,28,29]$. Here $A_{0}$ and $m_{0}$ are the common trilinear coupling and the scalar mass parameter, respectively. Fitting the CMSSM to the Higgs mass taking into account low energy and LHC constraints one finds that the best fit point [30] has the typical mass scales $m_{\tilde{g}}, m_{\tilde{q}} \gtrsim 2 \mathrm{TeV}, m_{\tilde{l}_{R}} \simeq 600 \mathrm{GeV}$ and $m_{\tilde{\chi}_{1}^{0}} \simeq 450 \mathrm{GeV}$. This implies, that the up-to now negative search results at the LHC are consistent with this part of the parameter which, however, will be probed by the current and next LHC runs. Also in even more general high scale models with non-universal parameter at $M_{G U T}$ the requirement of large trilinear couplings, e.g. $\left|A_{0}\right| \simeq(1-3) \max \left(M_{1 / 2}, m_{Q_{3}}, m_{U_{3}}\right)$, persists [31].

There is however a potential problem with large trilinear couplings such as $A_{t}$ or $A_{0}$; namely the danger that a global minimum of the scalar potential exists which is colour and/or charge breaking. It has been shown that large regions of the CMSSM parameter space with $m_{h} \simeq 125 \mathrm{GeV}$ are indeed ruled out by color/charge breaking minima [32].

High scale models like GMSB or CMSSM predict a rather hierarchical mass spectrum for the supersymmetric particles giving rise to hard jets and leptons at the LHC in combination with large missing transverse momentum with only small/tiny SM background. In contrast to these models, the general MSSM allows for the possibility that at least part of the spectrum with different species is rather compressed leading to substantial reduction of mass the bounds of various supersymmetric particles, see e.g. [14, 33, 34, 35]. This can be even more involved if flavour violating and/or CP violating parameters are present, which could still be large for the third generation [36, 37], as this can significantly alter the decay branching ratios.

A particular subclass of the general MSSM are so-called 'natural SUSY' scenarios [38, 39, 40]. The basic idea is of these models is to take only those SUSY particles close the electroweak scale which give a sizeable contribution sizeably to the Higgs mass $m_{h}$. In this way a too large finetuning of parameters of unrelated sectors is avoided. Multi-TeV scale masses are assigned to all other SUSY particles. In particular, the higgsinos, the partners of the Higgs bosons, and the light stop have masses of the order of a few hundred GeV. In addition the masses of the gluino and the heavier stop should be close to the TeV scale. This implies a rather compressed spectrum of the lightest neutralinos and the lightest chargino with mass differences of $O(1 \mathrm{GeV})$. This implies that the decay products of the leightest chargino and the second lightest neutralino are rather soft and cannot be identified as such at the LHC and are difficult to be detect in direct searches [41] at the LHC. This also implies that decays like $\tilde{b}_{1} \rightarrow \tilde{\chi}_{1}^{+} t$ and $\tilde{t}_{1} \rightarrow \tilde{\chi}_{1}^{0} t$ cannot be distinguished at the LHC. While these models are interesting from the point of view of fine-tuning they cannot explain the observed relic dark matter density as the annihilation cross sections of higgsinos are rather large[?]. Moreover, also this class of models requires large $A_{t}$ and, thus, the allowed parameter space gets constrained by the requirement of the non-existence of global charge and/or colour 
breaking minima [42]. As already mentioned above, data from the current LHC run imply mass bounds of up to $m_{\tilde{t}_{1}} \simeq 1 \mathrm{TeV}$ assuming a large mass hierarchy between the stop and the higgsinos $[43,44]$. We note for completeness that even in Natural SUSY the higgsinos might have larger masses due to possible existence of the soft SUSY breaking term [45] $\mu^{\prime} \tilde{H}_{u} \tilde{H}_{d}$ resulting in higgsino mass of order $\mu+\mu^{\prime}$.

Staying with MSSM particle content one can extend the model by allowing for R-parity violating couplings. Usually it is argued that this class is less motivated as it lacks a good dark matter candidate as the lighest supersymmetric particle (LSP) becomes unstable. However, supergravity implies that existence of the gravitino which is a potential dark matter candidate if it is light [46]. R-parity violation via lepton number violation gives a potential explanation of the observed neutrino data and mixing structure $[47,48]$. For the following remarks we concentrate on these parts of the parameter space. R-parity leads to a considerable change of the phenomenology due to the LSP decays. On the one side the amount of missing transverse energy is reduced on the other side there is possibility of displaced vertices which is a consequence of the smallness of the neutrino masses. Combining both aspects one finds that the LHC roughly is roughly the same as in the R-parity conserving MSSM once different channels are combined [49, 50]. Interestingly one can test if R-parity violation is indeed the source of neutrino masses and mixings as certain LSP decays are related to neutrino data, e.g. $\operatorname{BR}\left(\tilde{\chi}_{1}^{0} \rightarrow \tau q \bar{q}\right) / \mathrm{BR}\left(\tilde{\chi}_{1}^{0} \rightarrow \mu q \bar{q}\right) \simeq \tan ^{2} \theta_{\text {atm }}$ [51] with $\theta_{\text {atm }}$ being the atmospheric neutrino mixing angle. Such relations exist independent of the nature of the LSP [52].

\section{Extended supersymmetric models}

The requirement of having very large radiative corrections to explain $m_{h}$ is a hint to go beyond the MSSM. In non-minimal extensions, the tree-level bound can be pushed to larger values due to the extra $F$-contributions as in the next to minimal MSSM (NMSSM) [53] or due to extra $D$-term contributions in models with an enlarged gauge group [54] close to the electroweak scale. The main difference within the NMSSM is the extended Higgs sector but the phenomenology of the SUSY particles hardly changes. The main difference occurs in case of a singlino LSP as in this case the cascade decays of the SUSY particles get enlarged by an additional step. However, the main signatures remain as in the MSSM and we refer to ref. [55] for further details.

Here we concentrate on models with extended gauge groups. As examples we consider $S O(10)$ inspired left-right symmetric models, which have several virtues: (i) They gives an explanation of the observed neutrino masses and mixing pattern, (ii) They can explain the conservation of R-parity as $U(1)_{B-L}$ is a subgroup of $S O(10)$, (iii) The R-sneutrino, the partner of the right-handed neutrino, is a potential dark matter candidate $[56,57]$. In view of the Higgs mass, taking for example the breaking chain

$$
\begin{aligned}
S O(10) & \rightarrow S U(3)_{C} \times S U(2)_{L} \times S U(2)_{R} \times U(1)_{B-L} \\
& \rightarrow S U(3)_{C} \times S U(2)_{L} \times U(1)_{R} \times U(1)_{B-L} \cong S U(3)_{C} \times S U(2)_{L} \times U(1)_{Y} \times U(1)_{\chi}
\end{aligned}
$$

on obtains larger tree-level bounds such as [58] $m_{h} \leq m_{Z}^{2}+\frac{1}{4} g_{\chi}^{2} v^{2}$ where $g_{\chi} \simeq g_{Y}$ reducing the need for radiative corrections to about $50 \%$ which still is large. However, this reduces the need for rather 

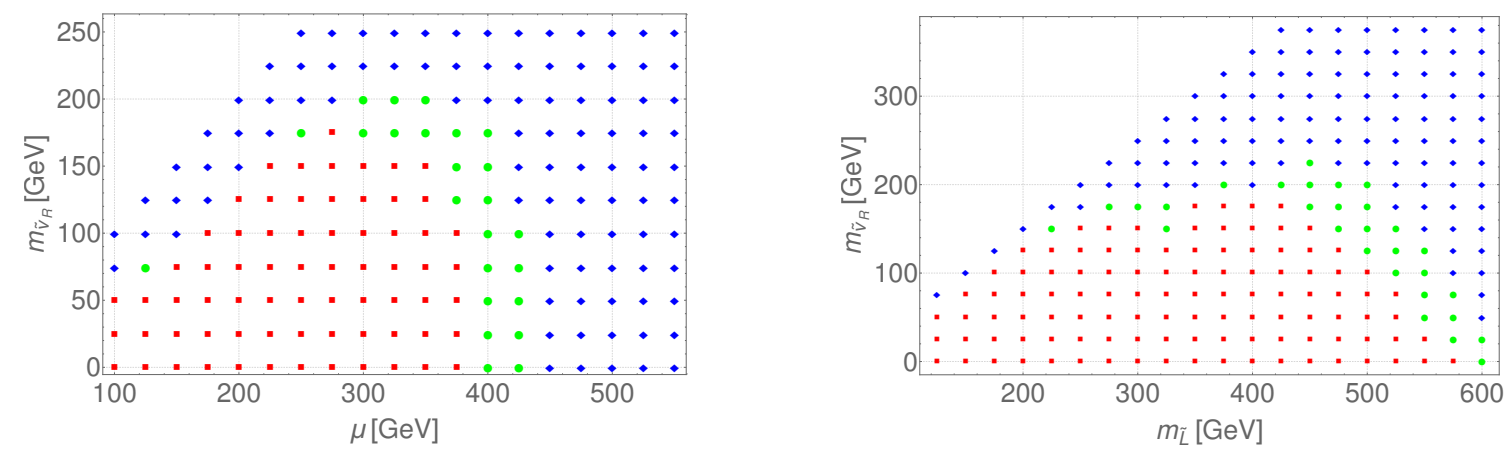

Figure 1: Left: LHC constraints on combinations of $m_{\tilde{v}_{R}}$ and $\mu$ due to chargino pair production $p p \rightarrow$ $\tilde{\chi}_{1}^{+} \tilde{\chi}_{1}^{-} \rightarrow l^{+} l^{-} \tilde{v}_{R} \tilde{v}_{R}^{*}$. Right: LHC constraints on combinations of $m_{\tilde{v}_{R}}$ and $m_{\tilde{L}}$ due to slepton/sneutrino production in case of an R-sneutrino LSP fixing $\mu=m_{\tilde{v}_{R}}+25 \mathrm{GeV}, M_{1}=M_{2}=2 \mathrm{TeV}, m_{v_{R}}=20 \mathrm{GeV}$ and $\tan \beta=6$. Red points are excluded, blue ones are allowed and in case of the green ones no conclusive statement can be drawn, within the known theoretical and experimental uncertainties. See ref. [62] for details.

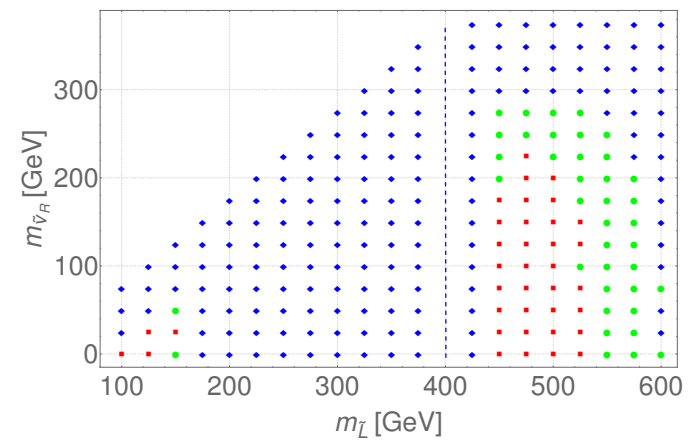

Figure 2: LHC constraints on combinations of $m_{\tilde{v}_{R}}$ and $m_{\tilde{L}}$ due to slepton/sneutrino production in case of an R-sneutrino LSP fixing $\mu=400 \mathrm{GeV}, M_{1}=M_{2}=2 \mathrm{TeV}, m_{v_{R}}=20 \mathrm{GeV}$ and $\tan \beta=6$. Red points are excluded, blue ones are allowed and in case of the green ones no conclusive statement can be drawn, within the known theoretical and experimental uncertainties. See ref. [62] for details.

large $A_{t}$ and thus the danger of charge/color breaking minima. The additional particle content has several phenomenological implications: (i) In particular in scenarios where a R-sneutrino is the lightest supersymmetric particle (LSP) one finds an enhanced lepton multiplicity in the cascade decays of supersymmetric particles [59]. Moreover, in contrast to the MSSM, where the the righthanded squarks decay essentially only into the LSP and a jet, one finds here sizeable branching ratios in the additional gaugino which decays further leading also in these case to cascade decays. While this reduces the importance of the 2-jet plus missing energy channel at the LHC, other channels become more important implying the current bounds on squarks and gluinos hardly change. (ii) The existence of a light additional, SM gauge singlet Higgs boson [54, 58, 60]. The direct production channles of these Higgs bosons is tiny but they show up in the decays of the heavier Higgs bosons as well of SUSY particles. (iii) Gauge kinetic mixing and additional $Z^{\prime}$ decay modes can significantly alter the LHC bounds on the $Z^{\prime}$ mass reducing it up to $500 \mathrm{GeV}[58,60]$.

One might ask if the additional particle content can potentially solve the dark matter problem 
of Natural SUSY. In principle, a light right-handed neutrino $v_{R}$ with a mass in the $\mathrm{keV}$ range can do this as in the $v \mathrm{SM}$ [61]. We note for completeness that this is rather difficult to achieve such a scenario in a simple $S O(10)$ scenario as the required smallness the neutrino Yukawa couplings can hardly be obtained. If this were the only change, then the LHC phenomenology of Natural SUSY would not change. It is well conceivable that the mechanism causing the lightness of the $v_{R}$ leads also to light sleptons and sneutrinos. Assuming that a $R$-sneutrino is the LSP one gets immediately constraints on the $\mu$-parameter from higgsino pair production as now the decay $\tilde{\chi}_{1}^{+} \simeq \tilde{h}_{1}^{+} \rightarrow l^{+} \tilde{v}_{R}$ is allowed [62]. Using 8-TeV and $13 \mathrm{TeV}$ (with an integrated luminosity $\mathscr{L}=13.9 \mathrm{fb}^{-1}$ ) LHC data one obtains a bound of about $380 \mathrm{GeV}$ on $\mu$ provided there is sufficient phase space. This bound is hardly improved by the $36 \mathrm{fb}^{-1}$ data [63]. In case that also the usual sleptons have masses in the range of a few hundred $\mathrm{GeV}$, they are mainly produced via $p p \rightarrow \tilde{l}_{L} \tilde{v}_{L}$. In such a scenario one gets constraints from LHC data on the soft SUSY breaking parameter $M_{\tilde{L}}$, which sets the mass scale of the sleptons, of up to $580 \mathrm{GeV}$ using the analyses implemented in the CheckMate package. We refer to ref. [62] for further details. Note, that these bounds apply to any other model containing the corresponding particles. The $36 \mathrm{fb}^{-1}$ data push this bound up to about $630 \mathrm{GeV}$ [63]. Here it has been assumed that the sleptons decay via two-body decays into charginos and neutralinos which decay further into higgsinos. In the part of parameter space, where the higgsinos are heavier than the sleptons three body decays such as $\tilde{l} \rightarrow q \bar{q} q^{\prime}$ occur. In case of the left-sneutrinos the decays into $h \tilde{v}_{R}$ and $Z \tilde{n}_{R}$ dominate if kinematically allowed. The combination of these decay modes implies a substantial reduction of the bounds on the sleptons down to about $200 \mathrm{GeV}$ [62] as can be seen in fig. 2. The main for this reductions are that the jets and leptons from the slepton decays are relativ soft and the appearance of the on-shell Higgs and Z-bosons in the sneutrino decays. This gets hardly changed even specific searches where Higgs bosons appear in the higgsino decays, e.g. searches for higgsinos decaying into bosons and a gravitino [64, 65], are taken into account [63].

\section{Conclusions}

Within the MSSM the explanation of the observed Higgs mass $m_{h} \simeq 125.1 \mathrm{GeV}$ requires large radiative correction. This can be either achieved via heavy stops and/or large left-right mixing in the stop sector. The latter can lead to charge/color breaking minima putting severe constraints on the corresponding parameter space. In high scale models such as CMSSM, NUHM or general GMSB, squarks and gluinos have masses in the 1-2 TeV range within the corresponding parameter regions which are currently probed at the LHC. If minimal GMSB were realised in nature then the observed Higgs mass requires a spectrum of coloured SUSY particles beyond the reach of LHC at $14 \mathrm{TeV}$.

In generic models with MSSM particle content the LHC bounds can be substantially reduced if the spectrum is at least partially compressed. However, if this realized in Nature, this will require a quite unusual pattern for supersymmetry breaking as the renormalisation group evaluation of the underlying parameters yields a quite hierarchical mass spectrum in unified models.

The relatively large value of $m_{h}$ can be understood as a hint to go beyond the MSSM. In extended models one can get additional tree-level contributions to $m_{h}$ due to F-terms, like in the NMSSM, and/or due to D-terms, like in models with extended gauge symmetries. This reduces somewhat the need for large radiative corrections. We have briefly sketched some important fea- 
tures of $S O(10)$ inspired models. Moreover, we have shown that in scenarios with an $R$-sneutrino LSP the LHC gives bounds on electroweakly produced particles of up to $580 \mathrm{GeV}$.

\section{References}

[1] G. Aad et al. [ATLAS Coll.], Phys. Lett. B 716 (2012) 1 [arXiv:1207.7214 [hep-ex]].

[2] S. Chatrchyan et al. [CMS Coll.], Phys. Lett. B 716 (2012) 30 [arXiv:1207.7235 [hep-ex]].

[3] M. Aaboud et al. [ATLAS Collaboration], Phys. Lett. B 784 (2018) 345 [arXiv:1806.00242 [hep-ex]].

[4] A. M. Sirunyan et al. [CMS Collaboration], JHEP 1711 (2017) 047 [arXiv:1706.09936 [hep-ex]].

[5] A. M. Sirunyan et al. [CMS Collaboration], [arXiv:1806.05246 [hep-ex]].

[6] M. Aaboud et al. [ATLAS Collaboration], arXiv:1808.08238 [hep-ex].

[7] A. M. Sirunyan et al. [CMS Collaboration], arXiv:1808.08242 [hep-ex].

[8] M. Aaboud et al. [ATLAS Collaboration], arXiv:1808.09054 [hep-ex].

[9] M. Aaboud et al. [ATLAS Coll.], JHEP 1709 (2017) 088 [arXiv:1704.08493 [hep-ex]].

[10] A. M. Sirunyan et al. [CMS Coll.], Phys. Rev. Lett. 119 (2017) no.15, 151802 [arXiv:1705.04673 [hep-ex]].

[11] A. M. Sirunyan et al. [CMS Collaboration], JHEP 1805 (2018) 025 [arXiv:1802.02110 [hep-ex]].

[12] M. Aaboud et al. [ATLAS Collaboration], arXiv:1808.06358 [hep-ex].

[13] H. Weber, talk at SUSY'18, 23-27 July 2018, Barcelona, Spain.

[14] T. J. LeCompte, S. P. Martin, Phys. Rev. D 85 (2012) 035023 [arXiv:1111.6897 [hep-ph]].

[15] U. Nierste, talk at ALPS 2018, April 15-20 2018, Obergurgl, Austria.

[16] M. Bauer and M. Neubert, Phys. Rev. Lett. 116 (2016) no.14, 141802 [arXiv:1511.01900 [hep-ph]].

[17] A. Crivellin et al., Phys. Rev. D 97 (2018) no.1, 015019 [arXiv:1706.08511 [hep-ph]].

[18] T. Faber et al., arXiv:1808.05511 [hep-ph].

[19] A. Crivellin, G. D’Ambrosio and J. Heeck, Phys. Rev. Lett. 114 (2015) 151801 [arXiv:1501.00993 [hep-ph]].

[20] A. Celiset et al., Phys. Rev. D 92 (2015) no.1, 015007 [arXiv:1505.03079 [hep-ph]].

[21] A. Falkowski et al., JHEP 1808 (2018) 061 [arXiv:1803.04430 [hep-ph]].

[22] A. Djouadi, Phys. Rept. 459 (2008) 1 [hep-ph/0503173].

[23] M. A. Ajaib et al., Phys. Lett. B 713 (2012) 462 [arXiv:1204.2856 [hep-ph]].

[24] M. Mangano (ed.), CERN Yellow Report CERN 2017-003-M [arXiv:1710.06353 [hep-ph]].

[25] S. Knapen, D. Redigolo, JHEP 1701 (2017) 135 [arXiv:1606.07501 [hep-ph]].

[26] M. Aaboud et al. [ATLAS Collaboration], Phys. Rev. D 98 (2018) no.3, 032008 [arXiv:1803.10178 [hep-ex]].

[27] H. Baer, V. Barger and A. Mustafayev, Phys. Rev. D 85 (2012) 075010 [arXiv:1112.3017 [hep-ph]].

[28] M. Kadastik et al., JHEP 1205 (2012) 061 [arXiv:1112.3647 [hep-ph]]. 
[29] O. Buchmueller et al., Eur. Phys. J. C 72 (2012) 2020 [arXiv:1112.3564 [hep-ph]].

[30] P. Bechtle et al., Eur. Phys. J. C 76 (2016) no.2, 96 [arXiv:1508.05951 [hep-ph]].

[31] F. Brummer, S. Kraml and S. Kulkarni, JHEP 1208 (2012) 089 [arXiv:1204.5977 [hep-ph]].

[32] J. E. Camargo-Molina et al., JHEP 1312 (2013) 103 [arXiv:1309.7212 [hep-ph]].

[33] S. Sekmen et al., JHEP 1202 (2012) 075 [arXiv:1109.5119 [hep-ph]].

[34] M. W. Cahill-Rowley et al., Phys. Rev. D 88 (2013) no.3, 035002 [arXiv:1211.1981 [hep-ph]].

[35] A. Arbey et al., Phys. Lett. B 720 (2013) 153 [arXiv:1211.4004 [hep-ph]].

[36] A. Bartl et al., Phys. Rev. D 70 (2004) 035003 [hep-ph/0311338].

[37] K. De Causmaecker et al., JHEP 1511 (2015) 125 [arXiv:1509.05414 [hep-ph]].

[38] C. Brust et al., JHEP 1203 (2012) 103 [arXiv:1110.6670 [hep-ph]].

[39] M. Papucci et al., JHEP 1209 (2012) 035 [arXiv:1110.6926 [hep-ph]].

[40] L. J. Hall, D. Pinner and J. T. Ruderman, JHEP 1204 (2012) 131 [arXiv:1112.2703 [hep-ph]].

[41] D. Barducci et al., JHEP 1507 (2015) 066 [arXiv:1504.02472 [hep-ph]].

[42] J. E. Camargo-Molina et al., Phys. Lett. B 737 (2014) 156 [arXiv:1405.7376 [hep-ph]].

[43] The ATLAS Coll., ATLAS-CONF-2017-020.

[44] K. Yoshihara [ATLAS Collaboration], arXiv:1709.01053 [hep-ex].

[45] G. G. Ross, K. Schmidt-Hoberg and F. Staub, JHEP 1703 (2017) 021 [arXiv:1701.03480 [hep-ph]].

[46] M. Hirsch, W. Porod and D. Restrepo, JHEP 0503 (2005) 062 [hep-ph/0503059].

[47] M. Hirsch et al., Phys. Rev. D 62 (2000) 113008 Erratum: [Phys. Rev. D 65 (2002) 119901] [hep-ph/0004115].

[48] A. Dedes, S. Rimmer and J. Rosiek, JHEP 0608 (2006) 005 [hep-ph/0603225].

[49] F. de Campos et al., JHEP 0805 (2008) 048 [arXiv:0712.2156 [hep-ph]].

[50] F. De Campos et al., Phys. Rev. D 82 (2010) 075002 [arXiv:1006.5075 [hep-ph]].

[51] W. Porod et al., Phys. Rev. D 63 (2001) 115004 [hep-ph/0011248].

[52] M. Hirsch and W. Porod, Phys. Rev. D 68 (2003) 115007 [hep-ph/0307364].

[53] U. Ellwanger and C. Hugonie, Mod. Phys. Lett. A 22 (2007) 1581 [hep-ph/0612133].

[54] M. Hirsch et al., JHEP 1202 (2012) 084 [arXiv:1110.3037 [hep-ph]].

[55] U. Ellwanger, C. Hugonie and A. M. Teixeira, Phys. Rept. 496 (2010) 1 [arXiv:0910.1785 [hep-ph]].

[56] S. Gopalakrishna, A. de Gouvea and W. Porod, JCAP 0605 (2006) 005 [hep-ph/0602027].

[57] C. Arina and N. Fornengo, JHEP 0711 (2007) 029 [arXiv:0709.4477 [hep-ph]].

[58] M. E. Krauss, W. Porod and F. Staub, Phys. Rev. D 88 (2013) no.1, 015014 [arXiv:1304.0769 [hep-ph]].

[59] M. Hirsch et al., Phys. Rev. D 86 (2012) 093018 [arXiv:1206.3516 [hep-ph]].

[60] M. E. Krauss et al., Phys. Rev. D 86 (2012) 055017 [arXiv:1206.3513 [hep-ph]]. 
[61] T. Asaka and M. Shaposhnikov, Phys. Lett. B 620 (2005) 17 [hep-ph/0505013].

[62] N. Cerna-Velazco et al., Eur. Phys. J. C 77 (2017) no.10, 661 [arXiv:1705.06583 [hep-ph]].

[63] N. Cerna-Velazco et al., in preparation.

[64] A. M. Sirunyan et al. [CMS Collaboration], Phys. Rev. D 97 (2018) no.3, 032007 [arXiv:1709.04896 [hep-ex]].

[65] M. Aaboud et al. [ATLAS Collaboration], [arXiv:1806.04030 [hep-ex]]. 